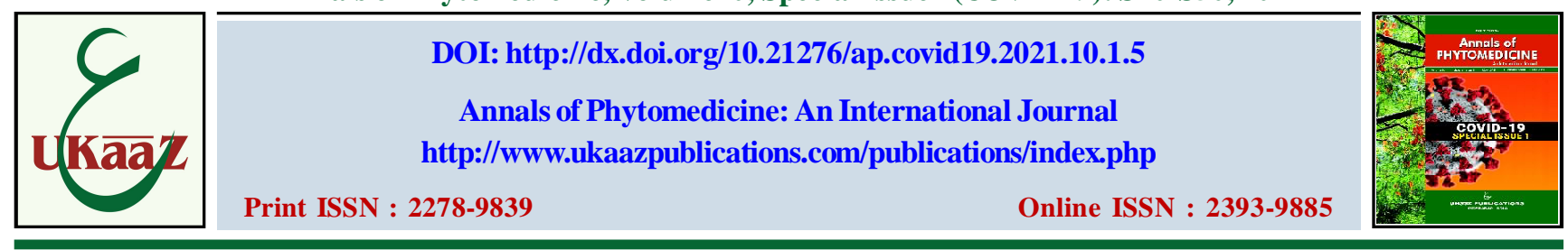

\title{
Indian red rice phenolic metabolites as potential natural inhibitors of SARS-CoV-2 main protease: A metabolomic and in silico study
}

\author{
Shrijana Rasaily, Ashrita C Haldipur and N. Srividya \\ Department of Food and Nutritional Sciences, Sri Sathya Sai Institute of Higher Learning, Deemed University, Anantapur-515134, A.P., India
}

\section{Article Info}

Article history

Received 8 April 2021

Revised 26 May 2021

Accepted 27 May 2021

Published Online 30 June 2021

\section{Keywords}

Eriodictyol

COVID-19

SARS-CoV-2

3-chymotrypsin like protease ADME

Molecular docking

Kuzhiadichan

\begin{abstract}
COVID-19 is a viral respiratory infectious disease caused by the novel strain of SARS-CoV-2 (Severe acute respiratory syndrome coronavirus 2 ). SARS-CoV-2 main protease $\left(\mathrm{M}^{\mathrm{pro}}\right)$ is associated with viral replication and transcription, thereby making it a potent target for combating COVID-19. Natural compounds from plants are being widely explored for viral inhibitors due to their safety aspect. Therefore, the present study aimed at predicting the antiviral property of red rice phenolic metabolites (RRPM) from three Indian rice varieties against SARS-CoV-2 $\mathrm{M}^{\text {pro }}$ through metabolomics and in silico studies. The Indian red rice cultivars, i.e., Veethi vadankan, Samba mosanam and Kuzhiadichan, were subjected to Q-TOF-LC-MS analysis and eighteen abundant polyphenols were selected using hierarchical cluster analysis. The abundant RRPM were virtually screened using Swiss ADME, a web-based tool. All the abundant polyphenols obeyed the Lipinski's rule except for hesperetin 3'-O-glucuronide, thereby exhibiting drug likeliness. Based on ADME analysis, fifteen of the RRPM showed good oral bioavailability, high GI absorption and acceptable overall bioavailability score. The 15 RRPM were further subjected to molecular docking analysis using Auto dock 1.5.6 against SARS-CoV-2M $\mathrm{M}^{\text {pro }}$. The analysis revealed 7, 4'-dihydroxy flavone and eriodictyol to have strong binding affinity $(>10 \mathrm{kcal} / \mathrm{mol})$ higher than nelfinavir $(-8.53 \mathrm{kcal} / \mathrm{mol})$. Eight other compounds also proved to be potential natural inhibitors of SARS-CoV-2 $\mathrm{M}^{\text {pro }}$. The present study has revealed Kuzhiadichan as a good source of eight potent natural inhibitors of SARS-CoV- $2 \mathrm{M}^{\text {pro }}$ and as a potential functional food for COVID-19 management.
\end{abstract}

\section{Introduction}

Coronavirus disease is a viral respiratory infectious disease caused due to the novel strain of SARS-CoV-2 (Severe acute respiratory syndrome coronavirus 2) which is responsible for symptoms like common cold, middle east respiratory syndrome and severe acute respiratory syndrome, etc. The pandemic has brought a serious health crisis affecting millions of people across the globe (Shah et al., 2020; Nupur, 2020). As per the recent updates provided by World Health Organization as on $21^{\text {st }}$ March 2021, around 223 countries have been found to be affected by COVID-19 disease with $1,22,524,424$ confirmed cases and the total number of confirmed deaths accounting up to 2,703,620 (WHO, 2021).

SARS-CoV-2 are positive stranded RNA viruses belonging to the family Coronaviridae, order Nidovirals and genus Beta coronavirus. The word 'corona' refers to 'crown', i.e., the virus particle having crown like structure (Singh et al., 2020). Technically, SARS- CoV-2 consists of 30000 nucleotides with the complex structure comprising of four major structural proteins, i.e., nucleocapsid protein, membrane protein, spike protein and envelope protein. The proteolytic processing of the SARS- CoV-2 proteins are responsible

Corresponding author: Dr. N. Srividya Associate Professor, Department of Food and Nutritional Sciences, Sri Sathya Sai Institute of Higher Learning, Deemed University, Anantapur-515134, A.P., India

E-mail: nsrividya@sssihl.edu.in

Tel.: +91-8554272567

Copyright $($ C) 2021 Ukaaz Publications. All rights reserved.

Email: ukaaz@yahoo.com; Website: www.ukaazpublications.com for transcription, translation, and replication of the virus. This process is mediated by the proteases - papain-like cysteine protease $\left(\mathrm{PL}^{\mathrm{pro}}\right)$ and 3 chymotrypsin like protease $\left(3 \mathrm{CL}^{\mathrm{pro}}\right)$ also called as the main protease $\left(\mathrm{M}^{\mathrm{pro}}\right)$. The main protease catalyzes the cleavage of the viral protein at multiple cleavage sites. Hence, SARS-CoV-2 $\mathrm{M}^{\text {pro }}$ is considered as a potent target for designing and formulating drugs against COVID-19 (Boopati et al., 2020).

Natural compounds from plants have a huge structural diversity and many of them possess drug like potency against certain diseases (Perez, 2003). Studies report that pharmacologically active compounds from plants are promising inhibitors of viral proteases with no toxic complications and are equally effective in regulating host immune response compared to the modern drugs (Paraiso et al., 2020; Bellik et al., 2020).

Among the phytochemicals, phenolic compounds present in different plants and foods have been reported to show defense mechanisms against viral pathogens like human immunodeficiency virus, poliovirus type 2 , hepatitis $\mathrm{B}$ and $\mathrm{C}$ virus, adenoviruses, influenza virus, etc. (Zakaryan, 2017). Recent studies are focusing on the possibilities of using phenolics from different plant and food sources in the management of COVID-19 pandemic (Chojnacka et al., 2020). Several pigmented rice varieties, i.e., red, purple, black, brown, etc., from countries such as China, Japan and Korea have been reported as good sources of phenolic compounds such as phenolic acids, flavonoids, anthocyanins, and their derivatives (Deng et al., 2013). Few recent studies have also reported the phenolic profile in some Indian pigmented rice varieties (Choudhury et al., 2020; Haldipur and Srividya, 2021). However, the phenolic 
profiles of several other Indian pigmented rice varieties remain unexplored. Moreover, the potent antiviral property of red rice phenolics against SARS-CoV-2 has not been investigated earlier to the best of our knowledge.

Therefore, in the present investigation, the phenolic profiling of three unexplored traditional Indian pigmented rice varieties: Veethi vadankan, Samba mosanam and Kuzhiadichan was carried out using an advanced metabolomic technique. The red rice phenolics identified were further screened for possible antiviral action against COVID19 by targeting the inhibition of SARS-CoV-2 $\mathrm{M}^{\text {pro }}$ through in silico studies.

\section{Materials and Methods}

\subsection{Sample procurement}

The three Indian red rice varieties, namely: Veethi vadankan (VV), Samba mosanam (SM) and Kuzhiadichan (KC) were procured from the farms of Tamil Nadu, India. The colored grains were cleaned free from dirt and foreign materials manually. ground, sieved through $0.5 \mathrm{~mm}$ mesh. The powdered samples were freeze dried (Lyodel freeze dryer, India), followed by vacuum packaging and stored at $20^{\circ} \mathrm{C}$ for further analysis. The experimental design of the study is shown in Figure 1.

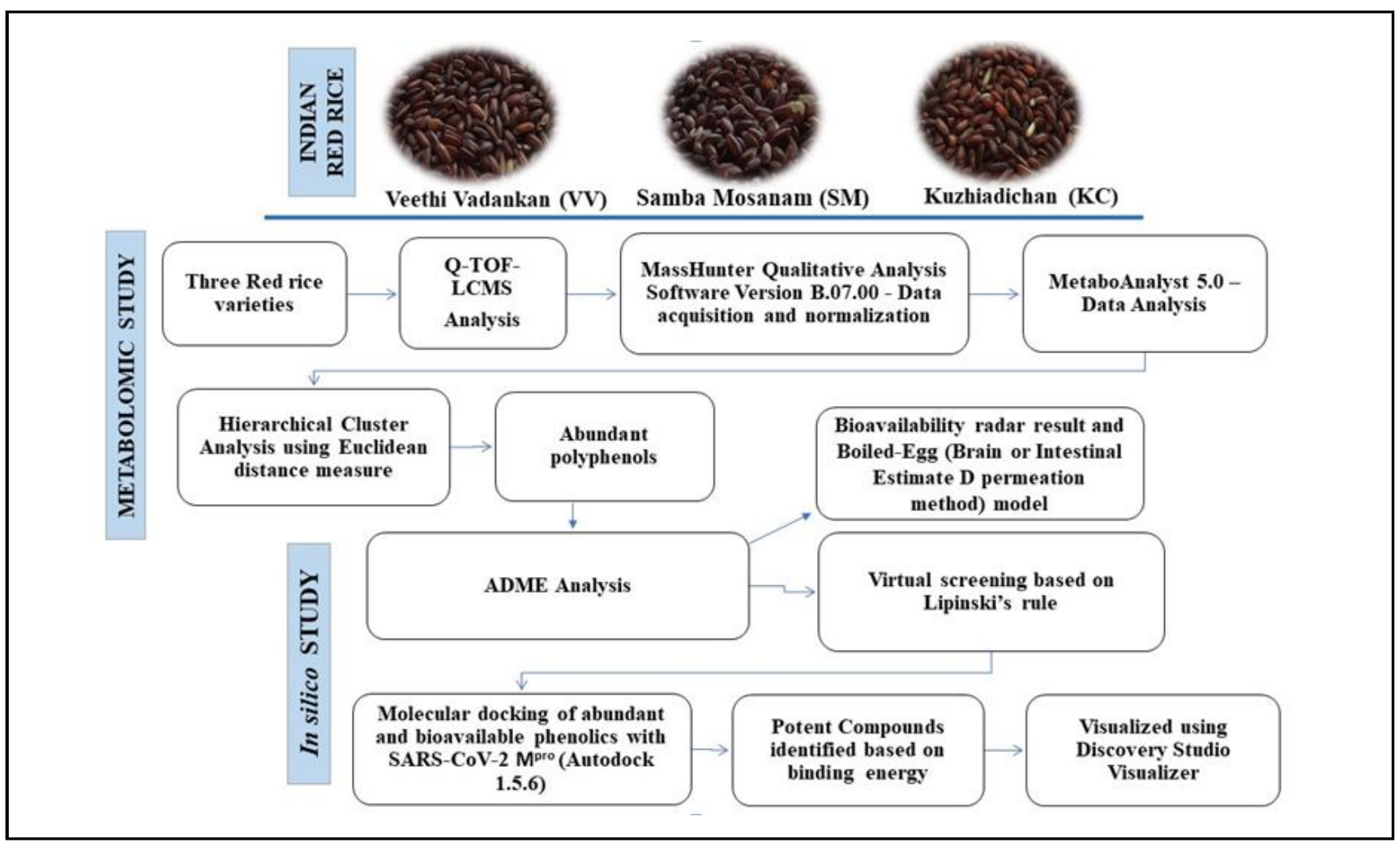

Figure 1: Experimental design of the study.

\subsection{Metabolomic analysis of red rice varieties}

\subsubsection{Extraction protocol}

Phenolic extraction was carried out by the method of Bordiga et al. (2014). Red rice flours $(0.1 \mathrm{~g})$ were extracted with $2.5 \mathrm{ml}$ of solvent mixture of methanol: water: formic acid (50:48:5:1.5 v/v). The mixture was ultrasonicated (Bransonic Digital Bath 5800, USA) for $10 \mathrm{~min}$ at $50^{\circ} \mathrm{C}$, followed by centrifugation (temperature maintained at $5^{\circ} \mathrm{C}$ ) (Sorvall, ST 8R, Thermo Fisher Scientific, Germany) at $2,500 \mathrm{~g}$ for $5 \mathrm{~min}$. Two more extractions were carried out from the resulting pellets. The extract aliquots were combined and concentrated using vacuum concentrator (miVac Duo concentrator, USA). The extracts were stored at $-20^{\circ} \mathrm{C}$ until analysis. The sample extracts were spiked with a mixture of $30 \mu \mathrm{l}$ of two internal standards: Epigallocatechin- 2,3,4- ${ }^{13} \mathrm{C}_{3}$ and Ferulic acid-1,2,3- ${ }^{13} \mathrm{C}_{3}(5 \mu \mathrm{g} / \mathrm{ml})$ for normalization and to combat any disparities during the ionization process and mass analysis.

\subsubsection{Instrument details and operating conditions}

The phenolic profiling of the red rice extracts was carried out using an analytical metabolomic platform: Q-TOF- LC-MS (Agilent 1290 Infinity LC system, Agilent Technologies, Santa Clara, CA, USA) provided with quaternary pump (G4204A), autosampler (G4226A), autosampler thermostat (G1330B) and column compartment thermostat (G1316C). The LC system was coupled to Agilent 6550 iFunnel Quadrupole Time-of-Flight (QTOF) with Agilent Jet Stream Thermal Gradient Technology.

The column involved in metabolites separation was Agilent Zorbax RRHD Eclipse XDB-C18 (2.1 x 100 mm, $1.8 \mu \mathrm{m}, 1200$ bar). The composition of mobile phases used were acetonitrile: (A), and 10 $\mathrm{mM}$ ammonium acetate in water adjusted to $\mathrm{pH} 5$ and formic acid (B). The operating parameters was as described earlier by Haldipur and Srividya (2021). 


\subsubsection{Data acquisition and analysis}

The data were obtained using an extended dynamic range mode (2 $\mathrm{GHz}$ ) and the full scan mass range of 50 to $1500 \mathrm{Da}$. Data acquisition and deconvolution was carried out using a software called as Agilent Mass Hunter Qualitative Analysis B.07.00 (Mass Hunter Qual, Agilent Technologies). ChemSpider, Phenol-Explorer, Pubchem and FoodB were used for obtaining the structures of phenolic compounds to create a custom phenolics database using Agilent Personal Compound Database and Library (PCDL). The database was uploaded in Mass Hunter Qual software's algorithm for data mining and analysis was further carried by using a freely available, streamlined metabolomics analysis tool- MetaboAnalyst 5.0.

\subsection{In silico studies}

\subsubsection{ADME analysis}

The ADME related parameters of the eighteen abundant red rice phenolic metabolites (RRPM) identified through metabolomics analysis were predicted using Swiss ADME (http://www.swiss adme.ch/), a free web - based tool. The RRPM were screened based on the Lipinski's rule (molecular weight $<500 \mathrm{Da}$, lipophilicity ( $\log \mathrm{P}$ ) value less than 5, H-bond donor $<5$ and $\mathrm{H}$-bond acceptor $<10$ ) for drug likeliness. The ADME analysis also predicts the oral bioavailability and yields an over all bioavailability score based on the six physicochemical properties, i.e., solubility, size, polarity, lipophilicity, flexibility, and saturation. The gastrointestinal absorption was predicted using the Boiled-Egg model (Daina et al., 2017).

\subsubsection{Molecular docking analysis}

Molecular docking is a useful in silico model to predict proteinligand interaction. The molecular interaction of fifteen abundant bioavailable RRPM with the viral protein, SARS-CoV-2 main protease $\left(\mathrm{M}^{\mathrm{pro}}\right)$ was analyzed using the software Auto dock 1.5.6. (Garg et al., 2020; Adem et al., 2020)

\subsubsection{Optimization of SARS-CoV-2 $\mathrm{M}^{\text {pro }}$ structure}

The crystalline structure of SARS-CoV-2 $\mathrm{M}^{\text {pro }}$, complexed with an inhibitor N3 with a resolution of $2.1 \AA$, was selected as a target protein for the study. The 3-dimensional structure of the protein with PDB ID: 6LU7 was retrieved in the PDB format form RCSB PDB database (https://www.rcsb.org/structure/6LU7). The protease consists of 306 amino acid. The optimization of protein structure was done prior to the actual docking which involved removal of the complexed inhibitor and water molecules, followed by the addition of Kollman charges, Gasteiger charges and assigning up of AD4 type atom, respectively.

\subsubsection{Optimization of phenolic ligands}

The abundant RRPM obeying Lipinski's rule, with good oral availability and bioavailability score were chosen as ligands for the study. The 3D structures of the compounds were obtained from PubChem database (https://pubchem.ncbi.nlm.nih.gov/) in SDF format which were later converted to PDB format using PyMOL, Schrodinger with the addition of H-bonds.

\subsubsection{Determination of active sites and docking}

The active sites of the optimized target protein (6LU7) were selected depending on the amino acid residues associated with it.
The amino acid residues involved in the active sites of the protein were selected based on the literature available: THR24, THR25, THR26, HIS41, MET49, PHE140, LEU141, ASN 142, GLY143, SER144, CYS145, HIS163, HIS164, MET165, GLU166, HIS172, ASP187, ARG188, GLN189 and THR190 (Jin et al., 2020). A grid of dimension $126 \times 78 \times 116$ with $0.375 \AA$ spacing was prepared around the active site of the protein to ensure that ligands fit into the active sites. While performing auto dock, the genetic algorithm was set as the search parameter, other docking parameters were set as default and the output file was obtained in DLG format utilizing Lamarckian genetic algorithm. Out of the 10 different protein confirmations, the one with the least binding energy ( $\mathrm{kcal} / \mathrm{mol}$ ) was selected for information on ligand efficiency, intermolecular energy, etc. PyMOL and BIOVIA discovery studio visualizer software packages were used to visualize the structure of the docked complexes and to understand the types of interactions involved.

\section{Results}

\subsection{Phenolic profiling of red rice varieties}

A total of seventy-nine metabolites were identified in the three red rice samples through Q-TOF- LC-MS analysis. The most abundant class of metabolites were flavonoids with twelve flavones eight isoflavonoids six flavanones five flavanols, four flavonols, two dihydroflavonols, and one anthocyanidin. About thirty-two phenolic acids were identified belonging to different subclasses such as hydroxycinnamic acid (15), hydroxybenzoic acid (9), hydroxyphenylpropanoic acid (2), hydroxyphenylacetic acid (3). Similarly, twelve other polyphenols were obtained from different subclasses, i.e., alkylphenols (1), hydroxybenzaldehyde (1), hydroxycinnamaldehyde (1), hydroxycoumarin (4) and hydroxyphenylpropene (1) and others were $p$-cresol, $o$-cresol, 2 methyl citric acid and malic acid.

\subsubsection{Relative quantification of phenolic metabolites}

The identified RRPM were subjected to hierarchical cluster analysis: Euclidean distance measure and Ward clustering algorithm using MetaboAnalyst 5.0 to generate a heatmap representing the top thirty phenolic compounds in the experimental samples (Figure 2). The dark red colored regions in the heat map represent the most abundant RRPM. Overall, eighteen such metabolites were identified.The abundant RRPM identified in Samba mosanam (SM) were 5, and included coumarin (other polyphenol), 3'-Omethylcatechin (flavanols), $p$-hydroxybenzoic acid (phenolic acid), gallic acid (phenolic acid) and dihydroxyphenylacetic acid (other polyphenol). Five RRPM were abundant in Veethi vadankan (VV), i.e., esculetin (other polyphenol), hesperetin 3'-O-glucuronide (flavanones), coumaroyl glucose (phenolic acid), ferulaldehyde and feruloylquinic acid (phenolic acids). The red rice varietyKuzhiadichan (KC) contained a more diverse range of phenolics with 14 abundant RRPM such as 2, 4 dihydroxybenzoic acid (phenolic acid), eriodictyol (flavanones), pinocembrin (flavanones), caffeic acid 4-O-glucoside (phenolic acid), 3'-hydroxyequol (isoflavonoids), 3'-hydroxydaidzein (isoflavonoids), 3'-hydroxyO-desmethylangolensin (isoflavonoids), 4-hydroxybenzaldehyde (other polyphenols), 7,4'-dihydroxyflavone (flavones), malic acid (other polyphenols) and ethyl gallate (phenolic acid). 


\subsection{ADME analysis}

The eighteen abundant RRPM were subjected to ADME analysis. RRPMs violating more than two parameters as per Lipinski's rule (hesperetin 3'-O-glucuronide) were ruled out from further analysis. Seventeen abundant RRPMs were found to obey Lipinski's rule, thereby exhibiting drug likeliness. The results obtained from ADME analysis are represented in Table 1, Figure 3 gives the bioavailability plots of the abundant RRPM identified in the experimental samples and those with high binding affinity. The compounds satisfying majority of the criteria, i.e., lipophilicity $(\operatorname{LogP})<5$, size $<500 \mathrm{~g} /$ mol, polarity (total polar surface area/TPSA) $<140 \AA$, flexibility (number of rotatable bonds) $<9$, insolubility $<6$, and insaturation (Csp3) < 1, shows good oral bioavailability (Krüger et al., 2020; Mahanthesh et al., 2020).

Table 1: ADME properties of the abundant red rice polyphenols

\begin{tabular}{|c|c|c|c|c|c|c|c|c|c|c|}
\hline \multirow[t]{2}{*}{ S.No. } & \multirow[t]{2}{*}{ Molecules } & \multicolumn{4}{|c|}{ Lipinski rule (Drug likeliness) } & \multirow{2}{*}{$\begin{array}{l}\text { TPSA* } \\
\text { (A) }\end{array}$} & \multirow{2}{*}{\multicolumn{2}{|c|}{$\begin{array}{l}\text { Water Solubility } \\
\text { LogS (ESOL) }\end{array}$}} & \multirow{2}{*}{$\begin{array}{l}\text { GI } \\
\text { Absor- } \\
\text { ption }\end{array}$} & \multirow{2}{*}{$\begin{array}{c}\text { Bioavai } \\
\text { lability } \\
\text { score }\end{array}$} \\
\hline & & $\begin{array}{c}\text { Mol } \\
\text { weight } \\
(<500 D a)\end{array}$ & $\begin{array}{c}\text { Lipo- } \\
\text { philicity } \\
(\log P<5)\end{array}$ & $\begin{array}{c}\text { H bond } \\
\text { donor } \\
(<5)\end{array}$ & $\begin{array}{c}\text { H-bond } \\
\text { Acceptor } \\
(<10)\end{array}$ & & & & & \\
\hline 1 & 3'-Hydroxydaidzein & 270.24 & 0.52 & 3 & 5 & 90.9 & -3.37 & Soluble & High & 0.55 \\
\hline 2 & 3'-Hydroxyequol & 258.27 & 1.62 & 3 & 4 & 69.92 & -3.49 & Soluble & High & 0.55 \\
\hline 3 & $\begin{array}{l}\text { 3'-Hydroxy-O-des- } \\
\text { methylangolensin }\end{array}$ & 274.27 & 1.1 & 4 & 5 & 97.99 & -3.61 & Soluble & High & 0.55 \\
\hline 4 & 3'-O-Methylcatechin & 304.29 & 0.49 & 4 & 6 & 99.38 & -2.57 & Soluble & High & 0.55 \\
\hline 5 & $\begin{array}{l}\text { 4-Hydroxybenzal } \\
\text { dehyde }\end{array}$ & 122.12 & 0.79 & 1 & 2 & 37.3 & -1.87 & $\begin{array}{l}\text { Very } \\
\text { soluble }\end{array}$ & High & 0.55 \\
\hline 6 & 7,4'-Dihydroxyflavone & 254.24 & 1.08 & 2 & 4 & 70.67 & -4.03 & $\begin{array}{l}\text { Moderately } \\
\text { soluble }\end{array}$ & High & 0.55 \\
\hline 7 & $\begin{array}{l}\text { Caffeic acid 4-O } \\
\text {-glucoside }\end{array}$ & 342.3 & -1.63 & 6 & 9 & 156.91 & -1.22 & Very soluble & Low & 0.11 \\
\hline 8 & Coumarin & 146.14 & 1.65 & 0 & 2 & 30.21 & -2.29 & Soluble & High & 0.55 \\
\hline 9 & Coumaroyl glucose & 472.44 & 0 & 5 & 10 & 170.82 & -2.92 & Soluble & Low & 0.55 \\
\hline 10 & $\begin{array}{l}\text { Dihydroxybenzoic } \\
\text { acid }\end{array}$ & 154.12 & 0.4 & 3 & 4 & 77.76 & -1.89 & Very soluble & High & 0.56 \\
\hline 11 & Eriodictyol & 288.25 & 0.16 & 4 & 6 & 107.22 & -3.26 & Soluble & High & 0.55 \\
\hline 12 & Esculetin & 178.14 & 0.45 & 2 & 4 & 70.67 & -2.28 & Soluble & High & 0.55 \\
\hline 13 & Ethyl Gallate & 198.17 & 0.49 & 3 & 5 & 86.99 & -2.01 & Soluble & High & 0.55 \\
\hline 14 & Gallic acid & 170.12 & -0.16 & 4 & 5 & 97.99 & -1.64 & Very soluble & High & 0.56 \\
\hline 15 & $\begin{array}{l}\text { Hesperetin 3'-O } \\
\text {-glucuronide }\end{array}$ & 478.4 & -1.72 & 6 & 12 & 192.44 & -3.39 & Soluble & Low & 0.11 \\
\hline 16 & Malic acid & 134.09 & -1.37 & 3 & 5 & 94.83 & 0.32 & $\begin{array}{l}\text { Highly } \\
\text { soluble }\end{array}$ & High & 0.56 \\
\hline 17 & p-Hydroxybenzoic acid & 138.12 & 0.99 & 2 & 3 & 57.53 & -2.07 & Soluble & High & 0.85 \\
\hline 18 & Pinocembrin & 256.25 & 1.27 & 2 & 4 & 66.76 & -3.64 & Soluble & High & 0.55 \\
\hline
\end{tabular}

*According to Veberet al., (2002), Topological Polar Surface Area (TPSA)< $140 \AA$ A indicates good oral bioavailability.

Fifteen RRPM was found to show good oral bioavailability and high gastrointestinal absorption (as given by Boiled-Egg model) Caffeic acid 4-O-glucoside and coumaroyl glucose were excluded from molecular docking studies due to their low oral bioavailability and gastrointestinal absorption. Bioavailability score was found to be highest for $p$-hydroxybenzoic acid (0.85), followed by 2,4 dihydroxybenzoic acid, gallic acid and malic acid, with a score of 0.56 . Twelve other compounds exhibited a bioavailability score of 0.55 and least value was recorded by caffeic acid 4-O-glucoside $(0.11)$.

\subsection{Molecular docking analysis}

The fifteen potent compounds obeying Lipinski's rule with good oral bioavailability and high GI absorption along with the control drug, nelfinavir were further subjected to molecular docking analysis to understand their inhibitory action against SARS-CoV-2 $\mathrm{M}^{\text {pro }}$. The binding affinity of these compounds with the protein were determined based on the values of binding energy $(\mathrm{kcal} / \mathrm{mol})$ obtained from Autodock software. The lesser the value of binding energy more is the binding affinity of the ligand with the target protein.

Docking analysis (Table 2) revealed that 7,4'-dihydroxyflavone $(-11.03 \mathrm{kcal} / \mathrm{mol})$ and eriodictyol $(-10.75 \mathrm{kcal} / \mathrm{mol})$ had stronger binding affinity with SARS-COV-2 $\mathrm{M}^{\text {pro }}$ compared to the control drug nelfinavir $(-8.53 \mathrm{kcal} / \mathrm{mol})$. Five other phenolic compounds (3'-hydroxyequol, 3'-O-methylcatechin, pinocembrin, 3'-hydroxydaidzein and 3'-hydroxy-O-desmethylangolensin) also showed a good binding affinity with the target protein with binding energy values ranging between $-6.57 \mathrm{kcal} / \mathrm{mol}$ to $-8.04 \mathrm{kcal} / \mathrm{mol}$. 
The docking poses and 2-D interactions of SARS-CoV-2 $\mathrm{M}^{\text {pro }}$ with 7,4'-dihydroxyflavone and eriodictyol are presented in Figure 4.

Among all the docked complexes, 7,4'-dihydroxyflavone exhibited highest binding affinity with the least binding energy of $-11.03 \mathrm{kcal} /$ mol. It was observed that five different bonds were involved in the interaction of protein and ligand complex. It formed hydrogen bonds with the active sites THR190, ASP187 and non-active site TYR54. It also formed a carbon hydrogen bond and a pication bond at the active sites GLU166 and HIS41, respectively. The phenolic metabolite formed an alkyl bond at the active site residue MET165 and weak Van der Waals interactions with seven other amino acids. Overall, it formed bonds with 8 active sites of SARS-CoV-2 $\mathrm{M}^{\text {pro }}$.

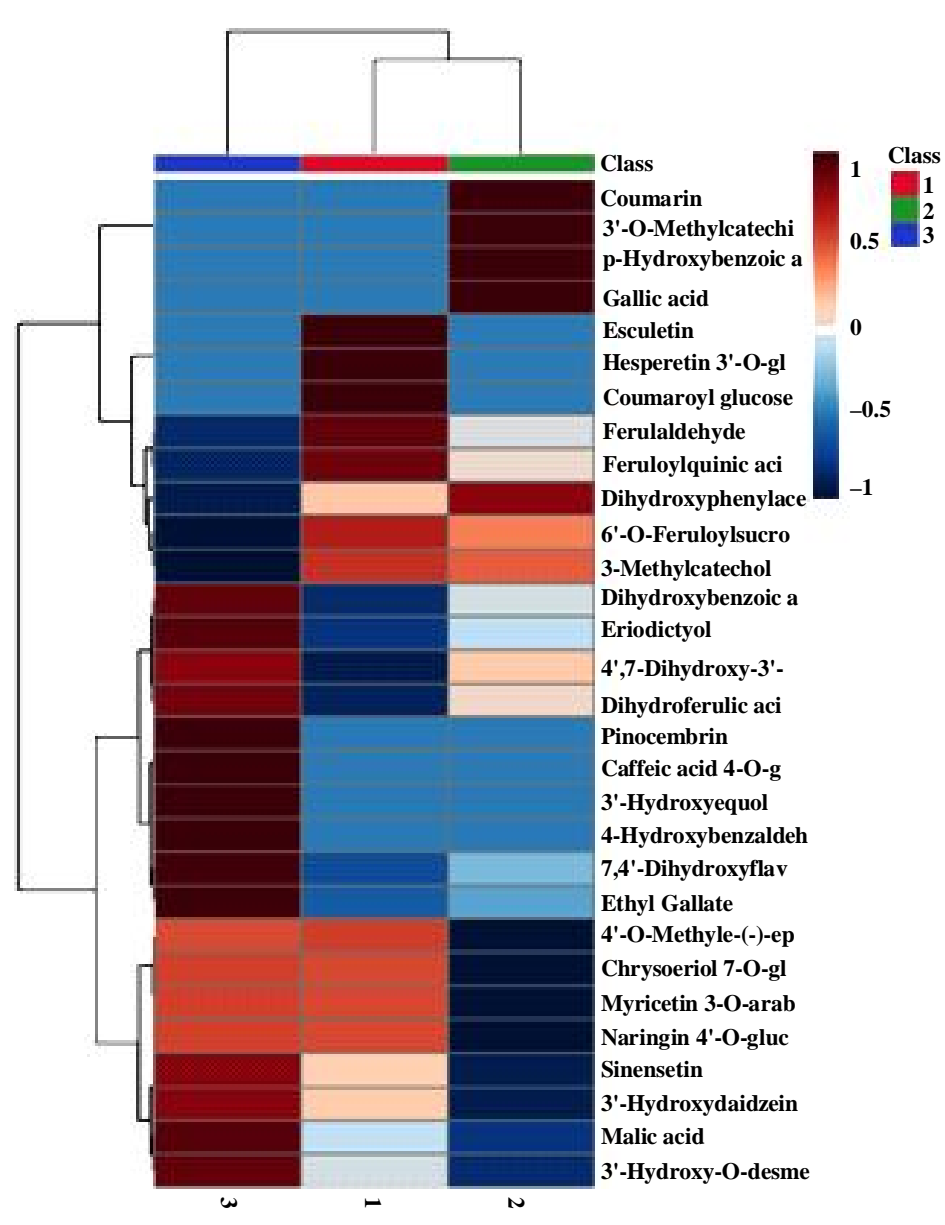

Figure 2: Heat map of top thirty phenolic compounds in red varieties (Class1: Veethivadankan, Class2: Samba Mosanam, Class 3: Kuzhiadichan).

Eriodictyol was also found to have a higher binding affinity $(-10.75$ $\mathrm{kcal} / \mathrm{mol}$ ), compared to the control drug. The structure of eriodictyol SARS-CoV-2 $\mathrm{M}^{\text {pro }}$ complex was stabilized by three different interactions, i.e., Van der Waals force, hydrogen bond and pication bonds. Hydrogen bonds were formed at the active site comprising the amino acid residues, THR190, ARG188, MET165, HIS41 and ASP187 of the protein. It also exhibited Van der Waals bonds with three active site residues MET49, GLU166 and HIS164. The ligand formed similar bonds with the amino acid residues TYR54, LEU167, PRO168, ALA191, VAL186 and GLN189. Eriodictyol was, thus found to bind with 11 active sites of SARS-CoV-2 $\mathrm{M}^{\text {Pro }}$.

Additionally, eight more RRPM, i.e., 3'-hydroxyequol, 3'-Omethylcatechin, pinocembrin, 3'-hydroxydaidzein, 3'-hydroxy-O- desmethylangolensin, esculetin, 4-hydroxybenzaldehyde and ethyl gallate also showed good binding potential with the target protein. The poses and interactions of the first five metabolites are represented in Figure 5 and described here.

3'-Hydroxyequol showed a good binding affinity $(-8.04 \mathrm{kcal} / \mathrm{mol})$ with the active sites of target protein by interacting with MET49, LEU141, GLY143, SER144, CYS145, HIS163, through hydrogen bonds. MET49 was found to interact with ligand through hydrogen, alkyl and pi-alkyl bonds. Additionally, pi-pi stacked bond along with pi-alkyl were observed between the ligand and HIS41 residue. Eight other amino acids can be observed forming weak Van der Waals interactions. An unfavorable donor-donor interaction was observed by GLN189 with the ligand molecule. 3'-Hydroxyequol formed bonds with a total of 15 active sites of SARS-CoV- $2 \mathrm{M}^{\text {pro }}$. 


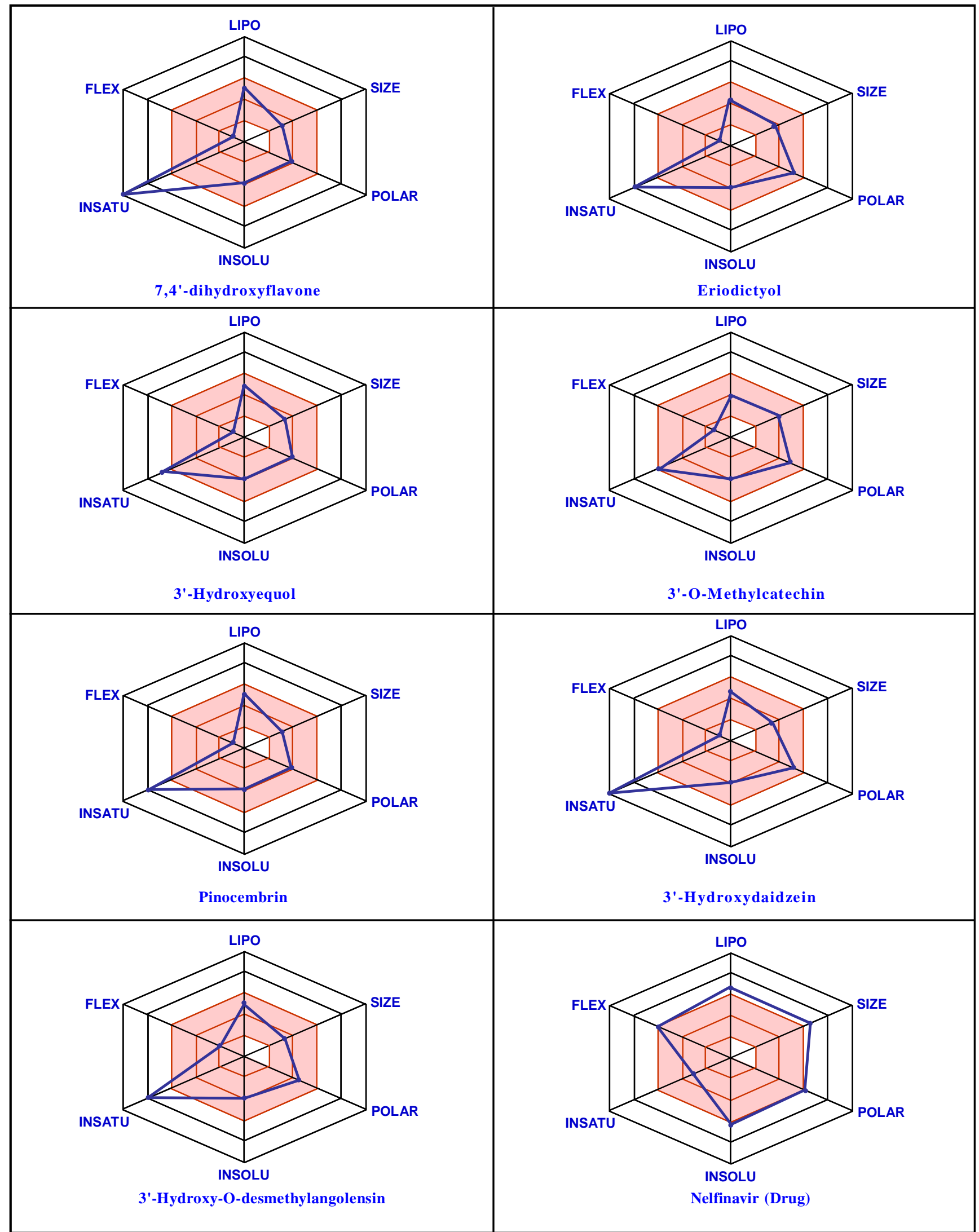

Figure 3: Bioavailability radar plots of nelfinavir (drug) and compounds exhibiting good inhibitory action against SARS-CoV-2 $\mathrm{M}^{\text {pro }}$. 


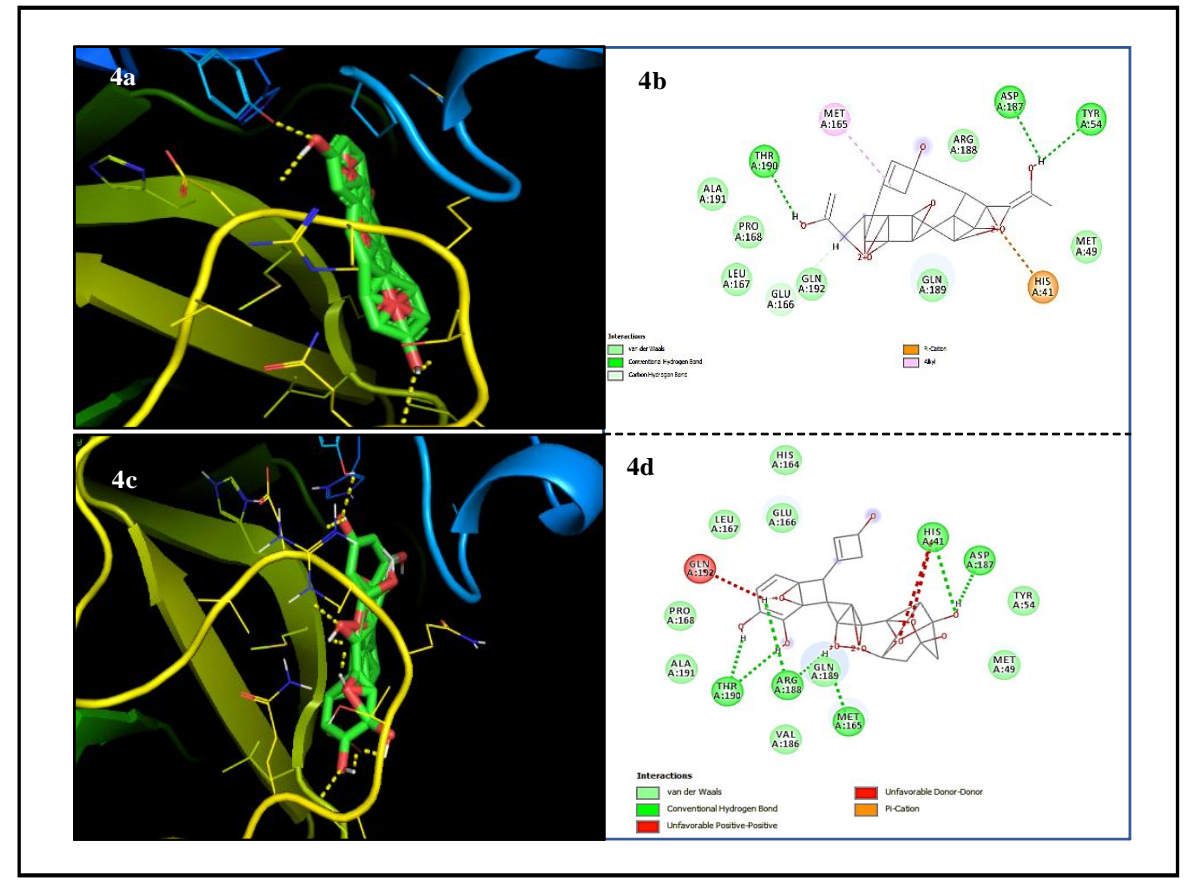

Figure 4:Molecular docking pose and 2-D view of interaction with SARS-CoV-M ${ }^{\text {pro }}$ of 7,4'-Dihydroxyflavone (Figures $4 \mathrm{a}$ and $\mathrm{b}$ ) and Eriodictyol (Figures $4 \mathrm{c}$ and d).

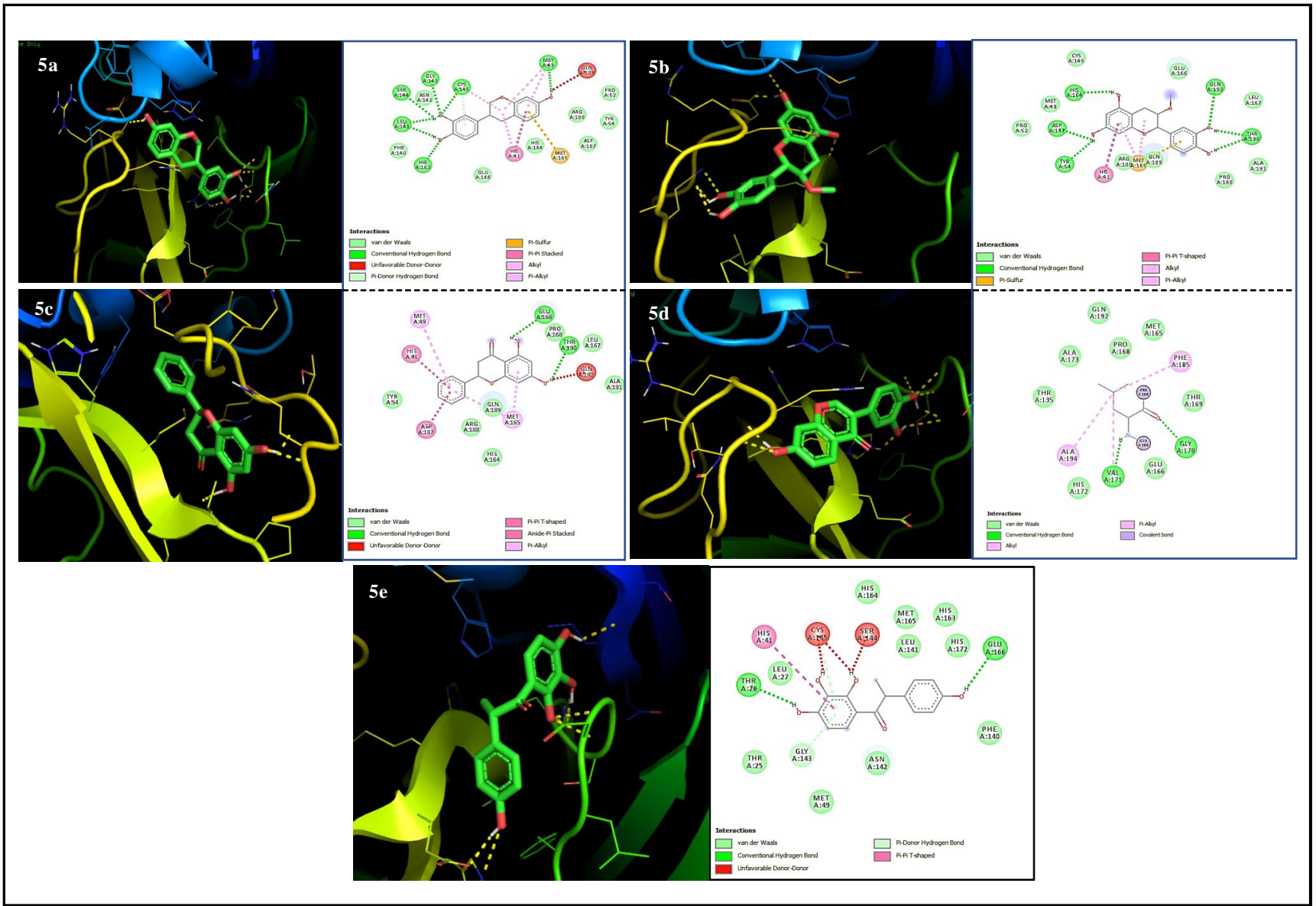

Figure 5: Molecular docking pose and 2-D view interaction of SARS-CoV-2 M $^{\text {pro }}$ with 5a, 3'-Hydroxyequol, 5b, 3'-OMethylcatechin, 5c, Pinocembrin, 5d, 3'-Hydroxydaidzein and 5e, 3'-Hydroxy-O-desmethylangolensin. 
Table 2: Molecular docking results of fifteen bioavailable abundant red rice polyphenols

\begin{tabular}{|c|c|c|c|}
\hline S. No. & Ligands & $\begin{array}{l}\text { Binding Energy } \\
\quad(\text { kcal } / \text { mol })\end{array}$ & $\begin{array}{l}\text { Interaction with the active sites of } \\
\text { SARS-CoV-2 MPro }\end{array}$ \\
\hline 1. & Nelfinavir (Control) & -8.53 & $\begin{array}{l}\text { MET49, ASN142, CYS145, MET165, GLU166, ASP187, } \\
\text { ARG188, GLN189, THR190 }\end{array}$ \\
\hline 2. & $\begin{array}{l}\text { 7,4'-Dihydroxyflavone } \\
(\mathrm{KC})^{\#}\end{array}$ & -11.03 & $\begin{array}{l}\text { HIS41, MET49, MET165, GLU166, THR190, ASP187, } \\
\text { ARG188, GLN189 }\end{array}$ \\
\hline 3. & $\begin{array}{l}\text { Eriodictyol } \\
(\mathrm{KC})\end{array}$ & -10.75 & $\begin{array}{l}\text { MET49, THR190, ARG188, HIS164, GLU166, MET165, } \\
\text { HIS41, ASP187, ARG188, GLN189, THR190 }\end{array}$ \\
\hline 4. & $\begin{array}{l}\text { 3'-Hydroxyequol } \\
\text { (KC) }\end{array}$ & -8.04 & $\begin{array}{l}\text { HIS41, PHE140, CYS145, ASN142, GLY143, SER144, } \\
\text { CYS145, LEU141, GLY143, HIS163, MET49, HIS164, } \\
\text { MET165, GLU166, ASP187 }\end{array}$ \\
\hline 5. & $\begin{array}{l}\text { 3'-O-Methylcatechin } \\
\text { (SM) }\end{array}$ & -7.96 & $\begin{array}{l}\text { HIS41, MET49, CYS145, HIS164, MET165, GLU166, } \\
\text { ASP187, ARG188, GLN189, THR190 }\end{array}$ \\
\hline 6. & $\begin{array}{l}\text { Pinocembrin } \\
\text { (KC) }\end{array}$ & -7.86 & $\begin{array}{l}\text { MET49, HIS41, HIS164 GLU166, THR190, ASP187, } \\
\text { ARG188, GLN189, THR190 }\end{array}$ \\
\hline 7. & $\begin{array}{l}\text { 3'-Hydroxydaidzein } \\
\text { (KC) }\end{array}$ & -7.29 & HIS172, GLU166, MET165 \\
\hline 8. & $\begin{array}{l}\text { 3'-Hydroxy-O-desmethylangolensin } \\
\text { (KC) }\end{array}$ & -6.57 & $\begin{array}{l}\text { HIS41, MET49, THR25, THR26, PHE140, LEU141, } \\
\text { GLY143, MET165, GLU166, HIS163, HIS164, HIS172 }\end{array}$ \\
\hline 9. & $\begin{array}{l}\text { Esculetin } \\
\text { (VV) }\end{array}$ & -5.81 & $\begin{array}{l}\text { ASN142, CYS145, GLY143, SER144, PHE140, LEU141, } \\
\text { HIS163, HIS164, MET165, GLU166, HIS172, GLN189 }\end{array}$ \\
\hline 10. & $\begin{array}{l}\text { Dihydroxybenzoic acid } \\
(\mathrm{KC})\end{array}$ & -5.39 & - \\
\hline 11. & $\begin{array}{l}\text { Coumarin } \\
\text { (SM) }\end{array}$ & -5.36 & - \\
\hline 12. & $\begin{array}{l}\text { 4-Hydroxybenzaldehyde } \\
\text { (KC) }\end{array}$ & -4.93 & HIS41, MET49, HIS164, MET165, ASP187, ARG188 \\
\hline 13. & $\begin{array}{l}\text { Ethyl Gallate } \\
(\mathrm{KC})\end{array}$ & -4.91 & MET165, GLU166, ASP187, ARG188, GLN189, THR190 \\
\hline 14. & $\begin{array}{l}\text { p-Hydroxybenzoic acid } \\
\text { (SM) }\end{array}$ & -4.89 & - \\
\hline 15. & $\begin{array}{l}\text { Gallic acid } \\
(\mathrm{SM})\end{array}$ & -4.75 & - \\
\hline 16. & $\begin{array}{l}\text { Malic acid } \\
(\mathrm{KC})\end{array}$ & -2.4 & - \\
\hline
\end{tabular}

\# The name of the red rice variety abundant in the respective polyphenol is mentioned in the parenthesis; KC-Kuzhiadichan; SM -Samba mosanam; VV -VethiVadakan. 
3'-O-methylcatechin is another ligand with good binding energy $(-7.96 \mathrm{kcal} / \mathrm{mol})$. Six different interactions were involved in stabilizing the docked complex through Van der Waals force, hydrogen bond, pi-sulfur, pi-pi T-shaped bond, alkyl, and pi-alkyl bond. The ligand formed hydrogen bonds at the active sites HIS164, ASP187,THR190 and non- active sites TYR54 and GLN192, respectively. The metabolite showed pi-sulfur, alkyl and pi-alkyl interactions at the active site MET165. The ligand was found to interact with nine other amino acids through Van der Waals force. Thus, 3'-O-methylcatechin formed bonds with a total of 15 active sites of SARS-CoV-2 $\mathrm{M}^{\text {pro }}$.

Pinocembrin $\mathrm{M}^{\text {pro }}$ complex had a binding energy of $-7.86 \mathrm{kcal} / \mathrm{mol}$ and exhibited five different interactions. The active sites, HIS41 and ASP187 coupled with the ligand through an amide-pi stacked bond and pi-pi T-shaped bond, respectively. Hydrogen bonds were formed with the active sites MET165,GLU166 and THR190. The ligand showed a pi-alkyl interaction with the active sites MET165 and MET49. Seven other amino acid residues were involved in the formation of Van der Waals bond with the ligand molecule. The complex also showed a donor-donor interaction with the GLN192 residue.

3'-hydroxydaidzein was observed to have a binding energy of -7.29 $\mathrm{kcal} / \mathrm{mol}$. A covalent bond interaction was observed with the active site GLU166 and another amino acid residue PRO168. The ligand shared two hydrogen bonds with GLY170 and VAL171. The structure was stabilized by alkyl and pi-alkyl bond through interaction with PHE185 and ALA194 residues, respectively. VAL171 was found to have shared both hydrogen bond and pi-alkyl bond with 3'hydroxydaidzein. It also formed bonds through Van der Waals force with eight other amino acid residues.

3'-hydroxy-O-desmethylangolensin was observed to have a binding energy of $-6.57 \mathrm{kcal} / \mathrm{mol}$. Altogether four different bonds were involved in the interactions -Van der Waals force, hydrogen bond, pi-donor hydrogen bond and pi-pi t-shaped bond. Hydrogen bonds were formed with the active sites THR26 and GLU166 and a pidonor hydrogen with another active site GLY143. Bonds due to weak Van der Waals were formed with ten other amino acid residues.The phenolic compound also formed a pi-pi T-shaped bond at the active site of HIS41.

\section{Discussion}

The present study demonstrated a great diversity in the composition of polyphenolic metabolites among the three Indian red rice varieties, VV, SM and KC. Flavonoids were the largest group of polyphenols present across all three red rice varieties, followed by phenolic acids and other polyphenols. A recent study conducted by Haldipur and Srividya (2020) also reported flavonoids to be the most abundant polyphenols in two other pigmented rice varieties (Bamboo rice and Garudan Samba). Similar polyphenols reported in the study were coumarin, dihydroquercetin, dihydroquercetin 3-O rhamnoside, pinocembrin, procyanidin dimer B, sinapic acidhexoside, naringin 4' -glucoside, 3-methoxynobiletin,esculetin, and isorhamnetin. Polyphenols like $p$-coumaric acid, ferulic acid, gallic acid, $p$-hydroxybenzaldehyde, and isorhamnetin identified in the experimental samples were also reported in pigmented rice varieties from Italy (Zaupa et al., 2015) and Thailand (Seekhaw et al., 2018). In a study by Kotamreddy et al. (2020), vanillic acid was reported to be absent in red rice varieties, similar to the observation in the current study.
Through, molecular docking analysis, it was found that several of the RRPM exhibited good binding potential at the active sites of the target protein $\mathrm{M}^{\mathrm{Pro}}$ indicating them to be potential antiviral agents against SARS-CoV-2.

Eriodyctiol showed a high binding potential with SARS-CoV-2 $\mathrm{M}^{\text {pro }}$ at eleven active sites. Eriodyctiol has been reported for the first time in the present study in pigmented rice metabolome. A glucoside of the compound, i.e., eriodyctiol-7-glucoside has been reported previously in Chinese brown rice genotypes (Dong et al., 2014). A recent study by Deshpande et al. (2020) also reported eriodyctiol with a good binding potential $(-6.7 \mathrm{kcal} / \mathrm{mol})$ against SARS-CoV-2 $\mathrm{M}^{\mathrm{Pro}}$.

7,4'-dihydroxyflavone exhibited the best binding affinity among all other RRPMs with interactions at eight active sites of SARS-CoV-2 $\mathrm{M}^{\text {pro }}$. No study till date has reported the antiviral activity of this metabolite.This is probably the first study to identify the antiviral potential of 7, 4'-dihydroxyflavone against COVID-19 virus. A structurally similar phenolic metabolite -3, 4'-dihydroxyflavone has been reported to show antiviral activity against influenza virus (Hossain et al., 2014).

Among eight other RRPM which exhibited antiviral activity against SARS-CoV-2, only one of them, i.e., esculetin have been reported earlier. Vijayaraj et al. (2020) reported the potential antiviral activity of an ester of esculetin identified in Marine Sponges against COVID-19 virus based on its binding potential with SARS-CoV-2 $\mathrm{M}^{\mathrm{Pro}}$. Few of these metabolites have been reported previously for activity against different viruses. Le et al. (2019) reported the antiviral activity of pinocembrin against Zika virus. 3'hydroxydaidzein has been reported to show antiviral activity against influenza virus (Özçelik et al., 2006).

The main antiviral RRPMs - 7,4'-dihydroxyflavone and eriodyctiol and other RRPMs with activity against SARS-CoV-2 $\mathrm{M}^{\text {Pro }}$ were found to be abundant in Kuzhiadichan rice variety. Interestingly, a recent study has shown strong negative correlation between rice consumption and the number of COVID-19 patients based on data obtained from nineteen countries from five continents (Watanabe and Inuma, 2020). A recent in silico study (Fujii, 2020) postulated that the micro RNA of rice (MIR2097-5p) can prevent COVID-19 by suppressing SARS-CoV-2 viral micro RNAs and HIPK2 target proteins.

Hence, this rice can be considered as a potential functional food in COVID-19 management.

\section{Conclusion}

The study showed that the three Indian red rice cultivars are rich sources of different bioactive phenolic metabolites. 7,4'dihydroxyflavone and eriodyctiol with high binding capacity on multiple active sites of SARS-CoV-2 target protein could be considered as potential candidates for antiviral drug development. The red rice variety-Kuzhiadichan, abundant in eight potential antiviral metabolites could be recommended as functional foods for COVID 19. Alternatively, the concentrate form of these phenolics could be used in the development of antiviral nutraceuticals which could act synergistically in the prevention and management of SARS-CoV-2 infection. Identification of the antiviral pharmacological action of 
these RRPM is an important milestone for scientists, health practitioners, and pharmacologists for future drug and functional food product development. Further, in vitro and in vivo studies are warranted for the validation of the antiviral activity of these red rice phenolic metabolites.

\section{Acknowledgements}

The authors acknowledge the founder Chancellor and the Management, Sri Sathya Sai Institute of Higher Learning, Andhra Pradesh, India, for providing research facilities in the Department of Food and Nutritional Sciences and the Central Research Laboratory, Anantapur, and in the Central Research Instruments Facility, Prasanthi Nilayam. The research was partially supported by the Indian Council of Medical Research (ICMR) through a project grant (No. 5/9/1205/2019-Nut) to the corresponding author.

\section{Conflict of interest}

The authors declare that there are no conflicts of interest relevant to this article.

\section{References}

Adem, S.; Eyupoglu, V.; Sarfraz, I.; Rasul, A.; Zahoor, A. F.; Ali, M.; Abdalla, Ibrahim, I. M. and Elfiky, A. A. (2020). Caffeic acid derivatives (CAFDs) as inhibitors of SARS-CoV-2: CAFDs-based functional foods as a potential alternative approach to combat COVID-19. Phytomedicine. Article ID 153310 (In Press).

Bellik, Y.; Becher-Bey, M.; Fatmi, W.; Kouidri, M.; Souagui, Y. and Selles, S. M.A (2020). Micronutrients and phytochemicals against COVID-19: Mechanism and molecular targets. Ann. Phytomed., 9(2):15-29.

Boopathi, S.; Poma, A. B. and Kolandaivel, P. (2020). Novel 2019 coronavirus structure, mechanism of action, antiviral drug promises and rule out against its treatment. J. Biomol. Struct. Dyn., https://doi.org/ 10.1080/07391102.2020.1758788.

Bordiga, M.; Gomez-Alonso, S.; Locatelli, M.; Travaglia, F.; Coïsson, J. D.; Hermosin- Gutierrez, I. and Arlorio, M.(2014). Phenolics characterization and antioxidant activity of six different pigmented Oryza sativa L. cultivars grown in Piedmont (Italy). Food Res. Int., 65:282-290.

Chojnacka, K.; Witek-Krowiak, A.; Skrzypczak, D.; Mikula, K. and Mlynarz, P. (2020). Phytochemicals containing biologically active polyphenols as an effective agent against Covid-19-inducing coronavirus. J. Funct. Foods, 73:1-8. Article ID 104146.

Choudhury, P.; Dutta, K. N.; Singh, A.; Malakar, D.; Pillai, M.; Talukdar, N. C.; Samantha, S. K. and Devi, R. (2020). Assessment of nutritional value and quantitative analysis of bioactive phytochemicals through targeted LC MS/MS method in selected scented and pigmented rice varietals. J. Food Sci., 85(6):1781-1792.

Daina, A.; Michielin, O. and Zoete, V. (2017). Swiss ADME: A free web tool to evaluate pharmacokinetics, drug-likeness, and medicinal chemistry friendliness of small molecules. Sci. Rep., 7(1):1-13.

Deng, G. F.; Xu, X. R.; Zhang, Y.; Li, D.; Gan, R. Y. and Li, H. B. (2013). Phenolic compounds and bioactivities of pigmented rice. Crit. Rev. Food Sci. Nutr., 53(3):296-306.

Deshpande, R. R.; Tiwari, A. P.; Nyayanit, N. and Modak, M. (2020). In silico molecular docking analysis for repurposing therapeutics against multiple proteins from SARS-CoV-2. Eur. J. Pharmacol., 886. Article ID 173430.

Dong, X.; Chen, W.; Wang, W.; Zhang, H.; Liu, X.and Luo, J. (2014). Comprehensive profiling and natural variation of flavonoids in rice. J. Integr. Plant Biol., 56(9):876-886.
Fujii Y.R. (2020). In silico study by quantum microRNA language for thedevelopment of anti-covid-19 agents: COVID-19 is preventedby rice MIR2097-5p through suppression of SARS-CoV-2 viralmicro RNAs plus HIPK2 target proteins.Virol. Immunol. J., 4(4), 000256.

Garg, S.; Anand, A.; Lamba, Y. and Roy, A. (2020). Molecular docking analysis of selected phytochemicals against SARS-CoV-2 M pro receptor. Vegetos, 33(4):766-781.

Haldipur, A. C. and Srividya, N. (2021). A comparative evaluation of in vitro antihyperglycemic potential of Bamboo seed rice (Bambusa arundinacea) and Garudan samba (Oryza sativa): An integrated metabolomics, enzymatic and molecular docking approach. J. Cereal Sci., 99:1-9. Article ID 103200

Hossain, M. K.; Choi, H. Y.; Hwang, J. S.;Dayem, A. A.; Kim, J. H.; Kim, Y. B. and Cho, S. G. (2014). Antiviral activity of 3, 4'-dihydroxyflavone on influenza a virus. J. Microbiol., 52(6), 521-526.

Jin, Z; Du, X.; Xu, Y.; Deng, Y.; Liu, M.; Zhao, Y.; Zhang, B.; Li, X.; Zhang, L.; Peng, C.; Duan, Y.; Yu, J; Wang, L.; Yang, K.; Liu, F.; Jiang, R.; Yang, X.; You, T.; Liu, X.; Yang, X.; Bai, F.; Liu, H.; Liu, X.; Guddat, L. W.; Xu, W.; Xiao, G.; Qin, C.; Shi, Z.; Jiang, H.; Rao, Z. and Yang, H. (2020). Structure of Mpro from SARS-CoV-2 and discovery of its inhibitors. Nature, 582(7811):289293.

Kotamreddy, J. N. R.; Hansda, C. and Mitra, A. (2020). Semi-targeted metabolomic analysis provides the basis for enhanced antioxidant capacities in pigmented rice grains. J. Food Meas. Charact., 14:1183-1191.

Krüger, A.; Maltarollo, G. V.; Wrenger, C. and Kronenberger, T. (2020). ADME profiling in drug discovery and a new path paved on silica. In: Drug Discovery and Development-New Advances, Editors: Gaitonde, V.; Karmakar, P. and Trivedi, A. In Tech Open, United Kingdom, pp:1-32.

Le Lee, J.;Loe, M. W. C.; Lee, R. C. H. and Chu, J. J. H. (2019). Antiviral activity of pinocembrin against Zika virus replication. Antiviral Res., 167, 13-24.

Mahanthesh, M. T.; Ranjith, D.; Raghavendra, Y.; Jyothi, R.; Narappa, G. and Ravi, M. V. (2020). Swiss ADME prediction of phytochemicals present in Butea monosperma (Lam.) Taub. J.Pharmacogn. Phytochem., 9(3):1799-1809

Vijayaraj, R.;Altaff, K.; Rosita, A. S.;Ramadevi, S.and Revathy, J. (2020). Bioactive compounds from marine resources against novel corona virus (2019-nCoV): In silico study for corona viral drug. Nat. Prod. Res., 9:1-5.

Nupur, M. (2020). Medicinal plants, aromatic herbs, and spices as potent immunity defenders: Antiviral (COVID-19) perspectives. Ann. Phytomed., 9(2):30-49.

Özçelik, B.; Orhan, I.and Toker, G. (2006). Antiviral and antimicrobial assessment of some selected flavonoids. Z. Naturforsch. C.J. Biosci., 61(9-10):632-638.

Paraiso, I. L.; Revel, J. S. and Stevens, J. F. (2020). Potential use of polyphenols in the battle against COVID-19. Curr. Opin. Food Sci., 32:149155 .

Perez, R. M. (2003). Antiviral activity of compounds isolated from plants. Pharm. Biol., 41(2):107-157.

Seekhaw, P.; Mahatheeranont, S.; Sookwong, P.; Luangkamin, S.; Neonplab, A. N. L. and Puangsombat, P. (2018). Phytochemical constituents of Thai dark purple glutinous rice bran extract [cultivar LuemPua (Oryza sativa L.)]. Chiang Mai J. Sci., 45(3):1383-1395.

Shah, B.; Modi, P. and Sagar, S.R. (2020). In silico studies on therapeutic agents for COVID-19: Drug repurposing approach. Life Sci., 252:112. Article ID 117652. 
Singh, P.; Tripathi, M. K.; Yasir, M.; Khare, R.; Tripathi, M. K. and Shrivastava, R. (2020). Potential inhibitors for SARS-CoV-2 and functional food components as nutritional supplement for COVID-19: A review. Plant Foods Hum. Nutr., 75(4):458-466.

Veber, D. F.; Johnson, S. R.; Cheng, H. Y.; Smith, B. R.; Ward, K. W. and Kopple, K. D. (2002). Molecular properties that influence the oral bioavailability of drug candidates. J. Med. Chem., 45(12):26152623.

Watanabe, S. and Inuma, K. (2020). Low COVID-19 infection and mortality in rice eating countries. Sch. J. Food Nutr., 3:326-8.
WHO (2021). Coronavirus disease (COVID-19) pandemic. Accessed on 21 st March 2021. https://www.who.int/emergencies/diseases/novelcoronavirus-2019.

Zakaryan, H.; Arabyan, E.; Oo, A. and Zandi, K. (2017). Flavonoids: Promising natural compounds against viral infections. Arch. of Virol., 162(9):2539-2551.

Zaupa, M.; Calani, L.; Rio, D. D.; Brighenti, F. and Pellegrini, N. (2015). Characterization of total antioxidant capacity and (poly) phenolic compounds of differently pigmented rice varieties and their changes during domestic cooking. Food Chem., 187:338-34

Shrijana Rasaily, Ashrita C Haldipur and N. Srividya (2021). Indian red rice phenolic metabolites as potential natural inhibitors of SARS- $\dot{C} 0 \mathrm{~V}-2$ main protease: A metabolomic and insilico study. Ann. Phytomed., Volume10, Special Issue1 (COVID-19): S40-S50. http://dx.doi.org/10.21276/ap.covid19.2021.10.1.5 